\title{
Lung cancer screening trials: The United States and beyond
}

\author{
Francine L. Jacobson, MD, MPH, ${ }^{\mathrm{a}}$ and Michael T. Jaklitsch, $\mathrm{MD}^{\mathrm{b}}$
}

The National Lung Screening Trial (NLST) has for the first time established a lung cancer-specific mortality benefit through screening using low-dose computed tomography (LDCT) of the chest. The $20 \%$ decrease in lung cancer-specific mortality and 7\% decrease in all-cause mortality found in the NLST begin a new chapter in the history of lung cancer. ${ }^{1}$ The success of surgical treatment for early-stage disease and the increasing number of targeted therapies will continue to change the prognosis of lung cancer in consort with early identification of the disease through screening, whether or not LDCT remains the initial screening test. The decrease in lung cancer deaths due to LDCT is necessary but not sufficient to establish computed tomography (CT) screening guidelines and associated clinical care. Secondary findings from the NLST will provide data regarding cost-effectiveness. Smaller trials in Europe are addressing important issues, including the organization and frequency of lung nodule follow-up. Although the demographics of lung cancer vary in different countries and trials vary somewhat in eligibility, data pooling for meta-analyses may provide additional power and insight.

\section{THE MAGNITUDE OF THE PROBLEM}

The United Nations Food and Agriculture Organization estimated 1.3 billion cigarette smokers in the world in $2010 .^{2}$ In developed countries where smoking has declined, former smokers remain at high risk for lung cancer. Lung cancer kills more than 1.3 million people annually. The high mortality reflects the late presentation of disease with metastases at time of diagnosis. Overall 5-year survival is $10 \%$ to $20 \%$, even in the United States, despite more than $70 \% 5$-year survival of stage 1 a disease. ${ }^{3}$

The growing cohort of lung cancer survivors is now 400,000. Empowering these survivors, who include individuals, particularly women, who have never smoked, promises to bring lung cancer into the open and lead screening for lung cancer as a prior generation led the way to screening

\footnotetext{
From the Division of Thoracic Imaging, ${ }^{a}$ Department of Radiology, and Division of Thoracic Surgery, ${ }^{\mathrm{b}}$ Department of Surgery, Brigham and Women's Hospital, Boston, Mass.

Disclosures: Authors have nothing to disclose with regard to commercial support. Presented at the 3rd International Minimally Invasive Thoracic Surgery Summit, Boston, Massachusetts, October 7-8, 2011.

Received for publication Oct 14, 2011; revisions received April 16, 2012; accepted for publication May 15, 2012; available ahead of print June 7, 2012.

Address for reprints: Francine L. Jacobson, MD, MPH, Division of Thoracic Imaging, Department of Radiology, Brigham and Women's Hospital, 75 Francis St, Boston,

MA 02115 (E-mail: fjacobson@ partners.org).

J Thorac Cardiovasc Surg 2012;144:S3-6

$0022-5223 / \$ 36.00$

Copyright (c) 2012 by The American Association for Thoracic Surgery

doi:10.1016/j.jtcvs.2012.05.027
}

for breast cancer. The health care system does not yet have the systems in place to provide lung cancer screening CT in the same manner as mammography despite the fact that lung cancer causes more deaths in women. Lessons from mammography, historical lung cancer screening studies, and ongoing research in Europe contribute to our understanding and will influence the progression of lung cancer screening from clinical trials into routine clinical practice.

\section{REQUIREMENTS FOR SCREENING}

For screening to be viable, 5 conditions must be met: (1) The disease must be common, (2) there must be effective treatment for the disease, (3) a sensitive test must be available, (4) earlier treatment must decrease disease-specific mortality, and (5) the test must be inexpensive and easily performed. Although CT scanning is inexpensive compared with medical care for more advanced lung cancer that is generally identified by symptoms, it is an order of magnitude more costly than most screening tests that are universally recommended. This requires selection of patients who have a particularly high risk of lung cancer through tobacco use and, to a lesser extent, environmental exposures and genetic predispositions. Patients who have had lung cancer and those who have a history of both smoking and asbestos exposure often receive surveillance CT scans. The use of clinical CT protocols, with radiation dose 3 to 5 or more times higher than LDCT protocols, should be reassessed in these patients who could have equal benefit with significantly lower radiation exposure. It is difficult to conceive of the expansion of lung cancer screening to those at low to moderate risk for lung cancer while CT scanning is the primary screening procedure. An inexpensive biomarker-based test that could be applied to blood or urine would be ideal for the more complete population screening that would be required to provide early identification of lung cancer in the increasing number of lifetime nonsmokers who develop lung cancer.

\section{PREVIOUS LUNG CANCER SCREENING STUDIES}

Many lessons have been learned from lung cancer screening trials during the past 50 years. The Mayo Clinic chest $\mathrm{X}$-ray (CXR) screening trial undertaken in the 1970s found no mortality benefit after accounting for the cause of death of every participant. This experience has informed study design decisions made in various LDCT screening trials, including the Early Lung Cancer Action Project (ELCAP) and the NLST. In 1999, the ELCAP reported the screening results for 1000 symptom-free volunteers who were at least 60 years of age with a smoking history of at least 10 
pack-years and no history of cancer other than nonmelanoma skin cancer. The ELCAP design was a single-arm study in which participants received both CXR and LDCT. Additional sites were added around the world creating the International ELCAP. From 1993 to 2005, 31,657 individuals at high risk for lung cancer were screened using LDCT for baseline and annual screening CT scans. The median age was 61 years with a median smoking history of 30 pack-years. Of the 484 lung cancers detected, $85 \%$ were stage I, and the 10-year survival of these patients was $88 \%$. Furthermore, 8 patients with stage I lung cancer identified in this trial who refused surgical therapy all died within 5 years. ${ }^{4}$ The NLST was started by the National Cancer Institute in 2002 as a randomized trial to compare 3 annual screenings by LDCT or CXR. There were 3 entry criteria: age 55 to 74 years, 30 pack-year smoking history, and smoking within the past 15 years. This trial (see below) was stopped early with a $20 \%$ reduction in lung cancerspecific mortality. ${ }^{1}$ For the first time, there was level 1 evidence that LDCT screening reduced lung cancer deaths.

It is difficult to prevent trial arms from becoming more similar, especially if $1 \mathrm{arm}$ is told to obtain routine care. The assignment of a specific routine level of care, such as used in the NLST with an annual CXR, resulted in significantly less crossover by participants seeking CT scans outside of the trial. ELCAP participants received both CT and CXR. This provided a valuable understanding of the potential for CT to identify early-stage lung cancer, although the single-arm study design precluded determination of mortality benefit. The experiences of ELCAP and contemporary Japanese investigators opened the pathway to the identification of early-stage lung cancers on CT scans. ${ }^{5-9}$

Along with the emergence of genetic mutation testing and targeted therapies, this has in turn supported the important reframing of the pathologic diagnosis of adenocarcinoma, now based on biomarkers rather than histologic pattern with lepidic growth.

\section{RANDOMIZED CLINICAL TRIALS}

The NLST randomized 53,454 individuals into 2 arms for 3 annual rounds of CT screening. Half of the participants received LDCT, and half of the participants received CXR. Screening was considered positive if the nodule was $4 \mathrm{~mm}$ or greater or suspicious lymphadenopathy or pleural effusion was present. Nodules that were stable for 2 years were reclassified as negative. In the LDCT group, 39.1\% had at least 1 positive screen; in the CXR group, $16 \%$ had at least 1 positive screen. Some $24.2 \%$ of screens were positive, of which $96.4 \%$ in the LDCT group and $94.5 \%$ in the CXR group were false-positives. All were followed for at least 5 years after the last round of screening. In the LDCT group, 1060 lung cancers were identified (645/ 100,000 person-years); in the CXR group, 941 lung cancers were identified (572/100,000 person-years). Of the 1060
TABLE 1. Lung cancer identified in the National Lung Screening Trial by stage

\begin{tabular}{lcl}
\hline Stage & $\begin{array}{c}\mathbf{6 4 9} \text { positive by } \\
\text { CT screen }\end{array}$ & $\begin{array}{c}\text { 279 positive by } \\
\text { CXR screen }\end{array}$ \\
\hline IA & $129 / 635(51.8 \%)$ & $90 / 275(32.7 \%)$ \\
IB & $71 / 635(11.2)$ & $41 / 275(14.9)$ \\
IIA & $26 / 635(4.1)$ & $14 / 275(5.1)$ \\
IIB & $20 / 635(3.1)$ & $11 / 275(4.0)$ \\
IIIA & $59 / 635(9.3)$ & $35 / 275(12.7)$ \\
IIIB & $49 / 635(7.7)$ & $27 / 275(9.8)$ \\
IV & $81 / 635(12.8)$ & $57 / 275(20.7)$ \\
\hline
\end{tabular}

$C T$, Computed tomography; $C X R$, chest x-ray.

lung cancers in the LDCT group, 649 were discovered by a positive screen; 44 lung cancers were screen negative, and 367 additional lung cancers were diagnosed between and after screens. Likewise, in the CXR group, 279 cancers were discovered by a positive screen; 137 were screen negative, and 525 additional lung cancers were diagnosed between and after screens. The LDCT group had 10\% less mortality, although the LDCT and CXR groups were comparable in regard to demographics and aggressive tumors unlikely to benefit from screening. The ability of CT scanning to identify nonsolid lesions and enlarged lymph nodes accounted for significant differences between the groups. The ability to decrease lung cancer deaths through early identification of disease is necessary but not sufficient for creating guidelines for lung cancer screening. In the CXR group, $30 \%$ of participants who presented later-stage lung cancer (stage III and IV) had positive CXR screening test results, whereas in the CT group, $43 \%$ of participants who presented with stage III and IV lung cancer had positive CT screening test results. Distribution of stage and histology is presented by group in Tables 1 and 2. This trial came to the simple but powerful conclusion that screening with LDCT reduces mortality from lung cancer. ${ }^{1}$

\section{EUROPEAN RANDOMIZED TRIALS}

The Dutch-Belgian Nederlands-Leuvens Longkanker Screening Onderzoek trial began the year after the NLST, in 2003, and is continuing to 2014. It is the largest European

TABLE 2. Lung cancer histology in the National Lung Screening Trial

\begin{tabular}{lcc}
\hline Histology & $\begin{array}{c}\text { 649 positive by } \\
\text { CT screen }\end{array}$ & $\begin{array}{c}\text { 279 positive by } \\
\text { CXR screen }\end{array}$ \\
\hline BAC (AIS) & $95 / 646(14.7 \%)$ & $13 / 276(4.7 \%)$ \\
Adenoca & $258 / 646(39.9)$ & $112 / 276(40.6)$ \\
Squamous & $136 / 646(21.1)$ & $70 / 276(25.4)$ \\
Large cell & $28 / 646(4.3)$ & $12 / 276(4.3)$ \\
NSCLC & $75 / 646(11.6)$ & $40 / 276(14.5)$ \\
Small cell & $49 / 646(7.6)$ & $28 / 276(10.1)$ \\
Carcinoid & $5 / 646(0.8)$ & $1 / 276(0.4)$ \\
\hline
\end{tabular}

$C T$, Computed tomography; $C X R$, chest x-ray; $B A C$, bronchioloalveolar carcinoma; $A I S$, adenocarcinoma in situ; NSCLC, non-small cell lung cancer. 
TABLE 3. Comparison of randomized computed tomography trials

\begin{tabular}{|c|c|c|c|c|}
\hline Trial & $\begin{array}{l}\text { Screening, } \\
\text { Rounds }\end{array}$ & Age (y) & $\begin{array}{l}\text { Pack-y } \\
\text { history }\end{array}$ & Quit (y) \\
\hline NLST & $\begin{array}{l}\text { LDCT vs } \\
\quad \text { CXR } \times 3\end{array}$ & $55-74$ & $\geq 30$ & $<15$ \\
\hline NELSON & $\begin{array}{l}\text { LDCT vs } \\
\quad \text { usual } \times 3\end{array}$ & $50-75$ & $\geq 15-18.75$ & $<10$ \\
\hline DLCST & $\begin{array}{l}\text { LDCT vs } \\
\quad \text { usual } \times 5\end{array}$ & $50-70$ & $\geq 20$ & $\begin{array}{c}<10 \text { after } \\
\text { age } 50 \mathrm{y}\end{array}$ \\
\hline DANTE & $\begin{array}{l}\text { LDCT vs } \\
\text { usual } \times 5 \\
\text { All baseline } \\
\text { CXR }\end{array}$ & $60-74$ men only & $\geq 20$ & $<10$ \\
\hline ITALUNG & $\begin{array}{l}\text { LDCT vs } \\
\quad \text { usual } \times 4\end{array}$ & $55-69$ & $\geq 20$ & $<10$ \\
\hline MILD & $\begin{array}{l}\text { LDCT vs } \\
\text { usual } \\
\text { Annual vs } \\
\text { biannual }\end{array}$ & $50-75$ & & $<10$ \\
\hline LUSI & $\begin{array}{l}\text { LDCT vs } \\
\quad \text { usual } \times 5\end{array}$ & $50-69$ & Heavy & No \\
\hline UKLS & $\begin{array}{l}\text { LDCT vs } \\
\text { usual (1) }\end{array}$ & $50-75$ & $5 \%$ risk in $5 y$ & Multivariable \\
\hline
\end{tabular}

$N L S T$, National Lung Screening Trial; $L D C T$, low-dose computed tomography; $C X R$, chest x-ray; NELSON, Dutch-Belgian Nederlands-Leuvens Longkanker Screening Onderzoek; DLCST, Danish Lung Cancer Screening Trial; DANTE, Detection and Screening of Early Lung Cancer by Novel Imaging Technology and Molecular Essays Trial; ITALUNG, CT RCT Tuscany; MILD, Multicentric Italian Lung Detection Trial (Milan); LUSI, German component of the European trial on the Efficacy of Multislice-CT Scan for the Early Detection of Lung Cancer; UKLS, UK Lung Screening Trial.

trial to date with 16,000 participants, although it is less than one third the size of NLST. This trial is randomizing participants to LDCT at baseline, 1 year, and 3 years to no screening. Subjects gain entry through a population-based enrollment. Questionnaires are used to judge the level of risk of developing lung cancer without absolute rules regarding smoking history. This allows a heterogeneous study group including nonsmokers with occupational exposures and a strong family history of lung cancer. ${ }^{10}$

Numerous smaller randomized trials are being conducted in Europe, including studies in Italy, France, and Denmark. The Danish Lung Cancer Screening Trial has randomized 4104 subjects to LDCT for 5 years or no screening. Entry criteria included 20 pack-year smoking history, forced expiratory volume in 1 second of $30 \%$ or more, and ages between 50 and 70 years. Although the current small size of the trial is limiting its current power, it has been designed in accordance with the Dutch-Belgian randomized lung cancer screening trial, the Nederlands-Leuvens Longkanker Screening Onderzoek, to allow pooling of the data from 20,000 individuals, providing $80 \%$ power to detect a $25 \%$ mortality reduction. Attention to nodule features, smoking cessation, and pulmonary nodule management are among the more specialized contributions being offered by the smaller trials. Differences in tobacco use patterns and other factors that vary from country to country also may provide insight. Meta-analyses will undoubtedly be performed, although screening guidelines may need to vary among countries because of such differences. A trial comparison is presented in Table 3.,10-14

\section{TRANSITION FROM CLINICAL TRIAL TO CLINICAL PRACTICE}

Low-dose CT screening trials have varied in subtle ways, such as (1) different entry criteria, (2) different nodule measurement strategies, and (3) different follow-up algorithms. The description of enrolled patients in the various trials will be more important in the meta-analysis of data than the entry criteria. CT scans in these clinical trials have been read primarily by subspecialty-trained thoracic radiologists working in academic centers. In some trials, dual reading and software tools for identification and measurement of lung nodules exceed the realistic resources that are available in community settings.

The International Association for the Study of Lung Cancer consensus statement recommending screening for individuals with the same characteristics as NLST participants provides the first basis for the logical extension of screening beyond the clinical trial, moving into clinical practice. ${ }^{1,10}$ At the current time, the decision to seek low-dose CT screening for lung cancer is an individual decision for which the patient and the physician and radiologist must share responsibility. Risk assessment is an integral part of this process. CT scanners and scanning protocols should follow the pattern established by the screening trials. This will result in low-dose radiation with high-quality images.

Population guidelines may need to be tailored to populations in different countries because of differences in smoking behavior and other risk factors for lung cancer, as well as financial constraints in the delivery of health care.

\section{QUESTIONS TO BE ANSWERED}

The Pisa Position Statement on March 4, 2011, listed 8 questions that need to be answered: (1) What is the optimal target population and how could individual risk be assessed to select a population at higher risk? (2) Could early detection biomarkers play a role in risk assessment? (3) What is the optimal management of suspicious nodules detected on CT? (4) What is the optimal screening protocol (age range, screen interval, number of screening rounds, value of recall CT scans)? (5) What is the effect of overdiagnosis? (6) What is the cost-effectiveness of CT screening? (7) What will CT screening add to an antismoking policy? (8) Is the efficacy of CT screening different in current versus former smokers? ${ }^{15}$

\section{CONCLUSIONS}

The largest randomized prospective trial using LDCT to screen for lung cancer, the NLST, has provided level 1 
evidence that screening reduces lung cancer mortality in 30 pack-year smokers between the ages of 55 and 74 years. LDCT screening for lung cancer will allow more patients to benefit from the highly successful treatments now available to treat most early-stage lung cancers. The interdisciplinary development and continuing evaluation of guidelines for screening, surveillance, and systematic nodule management will usher in a new era in the treatment of lung cancer.

\section{References}

1. The National Lung Screening Trial Research Team. Reduced lung-cancer mortality with low-dose computed tomographic screening. N Engl J Med. 2011; 365:395-409.

2. Food and Agriculture Organization of the United Nations. Higher world tobacco use expected by 2010 — growth rate slowing down; January 8, 2004. Available at: http://www.fao.org/english/newsroom/news/2003/26919-en.html. Accessed May 28, 2012

3. Howlader N, Noone AM, Krapcho M, Neyman N, Aminou R, Altekruse SF, et al, eds. SEER Cancer Statistics Review, 1975-2009(Vintage 2009 Populations). Bethesda, MD: National Cancer Institute. Available at: http://seer.cancer.gov/csr/ 1975_2009_pops09/. Accessed May 28, 2012.

4. The International Early Lung Cancer Action Program Investigators. Survival of patients with Stage I lung cancer detected on CT screening. N Engl J Med. 2006; 355:1763-71.

5. Kaneko M, Eguchi K, Ohmatsu H, Kakinuma R, Nanuke T, Suemasu K, et al. Peripheral lung cancer: screening and detection with low-dose spiral CT versus radiography. Radiology. 1996;201:798-802.
6. Sone S, Takashima S, Li F, Yang Z, Honda T, Maruyama Y, et al. Mass screening for lung cancer with mobile spiral computed tomography scanner. Lancet. 1998; 351:1242-5.

7. Henschke CI, McCauley DI, Yankelevitz DF, Naidich DP, McGuinness G, Miettinen OS, et al. Early lung cancer action project: overall design and findings from baseline screening. Lancet. 1999;354:99-105.

8. Marcus PM, Bergstralh EJ, Fagerstrom RM, Williams DE, Fontana R, Taylor WF, et al. Lung cancer mortality in the Mayo Lung Project: impact of extended follow-up. J Natl Cancer Inst. 2000;92:1308-16.

9. Swensen SJ, Jett JR, Harman TE, Midthun DE, Mandrekar SJ, Hillman SL, et al. CT screening for lung cancer: five-year prospective experience. Radiology. 2005; 235:259-65.

10. van Iersel CA, de Koning HJ, Draisma G, Mali WPTM, Scholten ET, Nackaerts K, et al. Risk-based selection from the general population in a screening trial: selection criteria, recruitment and power for the Dutch-Belgian randomized lung cancer multi-slice CT screening trial (NELSON). Int J Cancer. 2006;120:868-74.

11. Infante M, Lutman FR, Cavuto S, Brambilla G, Chiesa G, Passera E, et al. Lung cancer with spiral CT. Baseline results of the randomized DANTE trial. Lung Cancer. 2008;59:355-63.

12. Pedersen JH, Ashraf H, Dirksen A, Bach K, Hansen H, Toennesen P, et al. The Danish randomized lung cancer CT screening trial-overall design and results of the prevalence round. J Thorac Oncol. 2009;4:608-14.

13. Nair A, Hansell DM. European and North American lung cancer screening experience and implications for pulmonary nodule management. Eur Radiol. 2011; 21:2445-54.

14. Field JK, Smith RA, Aberle DR, Oudkerk M, Baldwin DR, Yankelevitz D, et al. International Association for the Study of Lung Cancer Computed Tomography Screening Workshop 2011 report. J Thorac Oncol. 2012;7:10-9.

15. International workshop on randomized lung cancer screening trials. State of the art in Europe after early conclusion of the US National Lung Screening Trial. The Pisa Position Statement. Pisa, Italy; March 4, 2011. 